\title{
Resonant Electric Dipole-Dipole Interactions between Cold Rydberg Atoms in a Magnetic Field
}

\author{
K. Afrousheh, P. Bohlouli-Zanjani, J. D. Carter, A. Mugford, and J. D. D. Martin \\ Department of Physics and Institute for Quantum Computing \\ University of Waterloo, Waterloo, ON, N2L 3G1, CANADA
}

(Dated: April 21, 2006)

\begin{abstract}
Laser cooled ${ }^{85} \mathrm{Rb}$ atoms were optically excited to $46 d_{5 / 2}$ Rydberg states. A microwave pulse transferred a fraction of the atoms to the $47 p_{3 / 2}$ Rydberg state. The resonant electric dipole-dipole interactions between atoms in these two states were probed using the linewidth of the two-photon microwave transition $46 d_{5 / 2}-47 d_{5 / 2}$. The presence of a weak magnetic field $\approx 0.5 \mathrm{G}$ reduced the observed line broadening, indicating that the interaction is suppressed by the field. The field removes some of the energy degeneracies responsible for the resonant interaction, and this is the basis for a quantitative model of the resulting suppression. A technique for the calibration of magnetic field strengths using the $34 s_{1 / 2}-34 p_{1 / 2}$ one-photon transition is also presented.

PACS numbers: 32.80.Rm, 34.20.Cf, 32.80.Pj
\end{abstract}

\section{INTRODUCTION}

The interactions between neighboring gas phase atoms can be enhanced by exciting them to Rydberg states. To obtain translationally cold interacting Rydberg gases $(\approx 100 \mu K)$, laser cooled atoms can be excited [1, 2]. The behavior of these cold Rydberg gases is often qualitatively different from that of hotter samples $(\approx 300 \mathrm{~K})$. For example, a cold cloud of Rydberg atoms can spontaneously evolve into a plasma under conditions in which a hot cloud could not [3]. Recent theoretical calculations suggest that cold interacting Rydberg atoms on uniformly spaced lattices will show properties analogous to that of a plasmon wave propagating along metal nanoparticles [4].

Translationally cold Rydberg atoms have recently received attention in the context of neutral atom quantum information processing. Theoretical work has suggested that Rydberg atom interactions may be used to implement gates between atoms holding qubits [5], for encoding qubits in multiatom clouds [6], and to implement "on demand" single-photon sources 7]. Recent experimental work has demonstrated a suppression of the average Rydberg density due to interactions - so called "local blockade" [8, 9], and rapid progress is expected in this area over the next several years [10].

Transitions between Rydberg states can be driven using microwave sources, which offer stability, linewidth, and modulation capabilities unrivaled by lasers. To elucidate the role of multibody interactions in cold Rydberg gases, Mourachko et al. 11] used microwave radiation to transfer a fraction of the optically excited Rydberg atoms into a different angular momentum state. Recently, Li et al. 12] have used a similar microwave redistribution pulse to establish the role of the electric dipole-dipole interaction in plasma formation from cold Rydberg gases. In addition, the linewidths of microwave driven transitions between Rydberg states can be used as a sensitive probe of interactions between Rydberg atoms [13].

Recently, we have probed the strong resonant electric dipole-dipole interactions between ${ }^{85} \mathrm{Rb}$ atoms in $45 d_{5 / 2}$ and $46 p_{3 / 2}$ states using the linewidth of a two-photon microwave transition from $45 d_{5 / 2}$ to $46 d_{5 / 2}$ [13]. The results were consistent with theoretical expectations, and this approach promises to be a powerful technique for studying cold Rydberg atom interactions.

How will weak magnetic fields $(\approx 0.5 \mathrm{G})$ influence the interactions between Rydberg atoms? Some of the energy degeneracies which are responsible for the resonant electric dipole-dipole interaction observed in Ref. 13] can be spoiled by application of a magnetic field. It may be desirable to excite neutral atoms to Rydberg states in a Ioffe-Pritchard trap without switching off the trapping fields. Rydberg atoms themselves could also be trapped using inhomogeneous magnetic fields [14].

In the present work we use microwave spectroscopy of Rydberg atoms to calibrate DC magnetic field strengths. Microwave fields are then used to manipulate Rydberg populations, and probe interactions, demonstrating that the dipole-dipole interaction between cold Rydberg atoms is partially suppressed by magnetic fields. A quantitative model is presented which explains these results.

\section{EXPERIMENTAL}

The apparatus is similar to that described in Ref. 13]. Cold ${ }^{85} \mathrm{Rb}$ atoms in a vapor-cell magneto-optical trap (MOT) 15 are excited to high- $n$ Rydberg states using a pulse of laser light. Microwave pulses are then used to transfer Rydberg population and probe interactions. The final state populations are analyzed by selective field ionization (SFI) 16]. The significant differences between the present apparatus and that in Ref. [13] are the laser excitation source and the control of the magnetic field during the experiment.

Instead of a pulsed dye laser system, a frequencydoubled cw Ti:sapphire system is used to excite Rydberg states. A commercial Ti:sapphire ring laser (Coherent MBR-110) operating at approximately $960 \mathrm{~nm}$ is 
frequency doubled in an external ring resonator (Coherent MBD-200) to produce approximately $90 \mathrm{~mW}$ at 480 $\mathrm{nm}$. Light pulses of $1 \mu \mathrm{s}$ duration are produced using an acousto-optic modulator. After the acousto-optic modulator the beam is coupled into a single mode fiber. The output beam from the fiber is collimated then focused and directed towards the trapped atoms. The highest Rydberg densities $\left(\approx 2 \times 10^{7} \mathrm{~cm}^{-3}\right)$ in this paper were obtained with optical powers of approximately $25 \mathrm{~mW}$.

Excitation of cold atoms to Rydberg states occurs as a two-color, two-photon process with the $480 \mathrm{~nm}$ light, and the nearly resonant, red detuned $780 \mathrm{~nm}$ light used for cooling and trapping [17]. Unfortunately, there are several atmospheric water absorption lines in the vicinity of $960 \mathrm{~nm}$ which restrict performance of the Ti:sapphire laser system. To avoid one of these, we excite $46 d_{5 / 2}$ states instead of the $45 d_{5 / 2}$ states used in Ref. [13].

Microwave horns are aimed at the Rydberg atoms through a fused silica window. To drive the $46 d_{5 / 2}-$ $47 d_{5 / 2}(\approx 35.7 \mathrm{GHz})$, the $46 d_{5 / 2}-47 p_{3 / 2}(\approx 22.1 \mathrm{GHz})$, and the $46 d_{5 / 2}-45 f_{5 / 2}(\approx 24.1 \mathrm{GHz})$ transitions we directly use the unamplified output of Agilent E8254A microwave synthesizers. For the $46 d_{5 / 2}-46 p_{3 / 2}(\approx$ $100.1 \mathrm{GHz})$ and the $34 s_{1 / 2}-34 p_{1 / 2}(\approx 104.1 \mathrm{GHz})$ transitions both an active quadrupler (Spacek Labs $\mathrm{P} / \mathrm{N}$ A100-4XW) or a passive tripler (Pacific Millimeter P/N $\mathrm{W} 3 \mathrm{WO}$ ) were used. These multipliers were driven by an Agilent E8254A synthesizer.

Two non-magnetic stainless steel electrode plates, separated by $36 \mathrm{~mm}$, are located to either side of the trapped atoms. These electrodes are used for SFI of Rydberg atoms [16]. The resultant ions are drawn by the same fields through a $6 \mathrm{~mm}$ hole in one of the electrode plates towards a microchannel plate detector. These plates are also useful for controlling the electric field in the experimental region. By varying the relative voltage difference of the two plates, their average voltage and the voltage of the $\mathrm{Rb}$ dispenser source with respect to the grounded chamber, it is possible to compensate for stray electric fields in all three directions in the experimental region. Stray electric fields are measured using the Stark shifts of a one-photon microwave transition 18. The residual electric field is estimated to be less than $0.1 \mathrm{~V} / \mathrm{cm}$.

To obtain estimates of the Rydberg density it is necessary to know the number of Rydberg atoms and their spatial extent. The spatial profile of the $480 \mathrm{~nm}$ beam determines the extent of the Rydberg cloud in two directions (the focus of this beam is much smaller than the trapped atom dimensions). Beam characterization cannot easily be done within the vacuum chamber. Instead, the rigidly attached output coupler and focusing assembly are moved to an optical table and scanning knife edge beam profile measurements are performed throughout the focal region (see for example Ref. [19]). The beam profile is Gaussian. Since there are collision processes which depend on the Rydberg density, the location of the tightest focus in the trap can be determined by moving the focusing lens relative to the trap to max- imize these processes. Once this location is found, the excitation beam profile can be systematically varied by moving the focusing lens. The beam was set to have a full width at half maximum (FWHM) of $0.190 \pm 0.015 \mathrm{~mm}$ at the location of the trap.

The extent of the Rydberg cloud in the third dimension is determined by the trapped atom profile. By scanning the direction of the $480 \mathrm{~nm}$ excitation laser - moving the beam over the trapped atom cloud - it is observed that there is an excellent correlation between the Rydberg signal and 780nm fluoresence imaging measurements of cloud extent (accounting for the finite width of the $480 \mathrm{~nm}$ beam). Therefore $780 \mathrm{~nm}$ fluorescence imaging gives the spatial extent of the Rydberg cloud in the direction of the $480 \mathrm{~nm}$ beam propagation. This is typically $0.5 \pm 0.1 \mathrm{~mm}$ (FWHM).

The beam profile of the $480 \mathrm{~nm}$ Rydberg excitation laser and the spatial profile of the trapped atoms are combined to convert the observed field ionization signals into spatially averaged Rydberg densities. If the waist of the excitation laser is significantly smaller than the width of the trap, and this waist does not change appreciably as the beam passes through the trap (conditions that are approximately satisfied in the present setup) the spatially averaged Rydberg density corresponding to a total number of Rydberg atoms $N_{R y d}$ is

$$
n_{R y d}=\left(\frac{2 \ln 2}{\pi}\right)^{3 / 2} \frac{N_{R y d}}{\left(\mathrm{FWHM}_{\perp}\right)^{2}\left(\mathrm{FWHM}_{\|}\right)}
$$

where $\mathrm{FWHM}_{\perp}$ is the full width at half maximum of the irradiance profile of the $480 \mathrm{~nm}$ light, and $\mathrm{FWHM}_{\|}$corresponds to the $5 p_{3 / 2}$ spatial distribution in the direction of the $480 \mathrm{~nm}$ beam.

Determination of $N_{R y d}$ requires knowledge of the absolute detection efficiency and gain of the microchannel plate detector (MCP). The absolute detection efficiency of an MCP depends on the kinetic energy at impact, the species involved, and surface conditions 20. A recommended efficiency for our specific conditions was not available. Based on information from the manufacturer and the literature [20] we estimate an initial detection efficiency of $50 \%$ - however this could be as high as $80 \%$ or as low as $20 \%$. The gain of the MCP was calibrated by observing the average total charge due to single events at higher bias voltages than normal operating conditions. To determine gain at the operating bias voltage, a series of measurements were taken at constant charged particle signal level with variable bias voltages.

The average densities quoted in this paper are obtained using Eq. 11. The uncertainty in these is dominated by $N_{R y d}$, which is limited by the initial detection efficiency estimate. 
a)

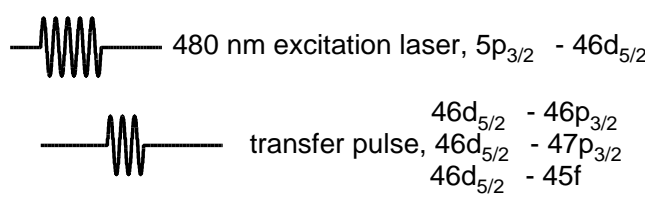

probe pulse

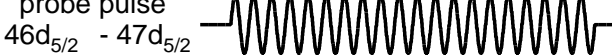
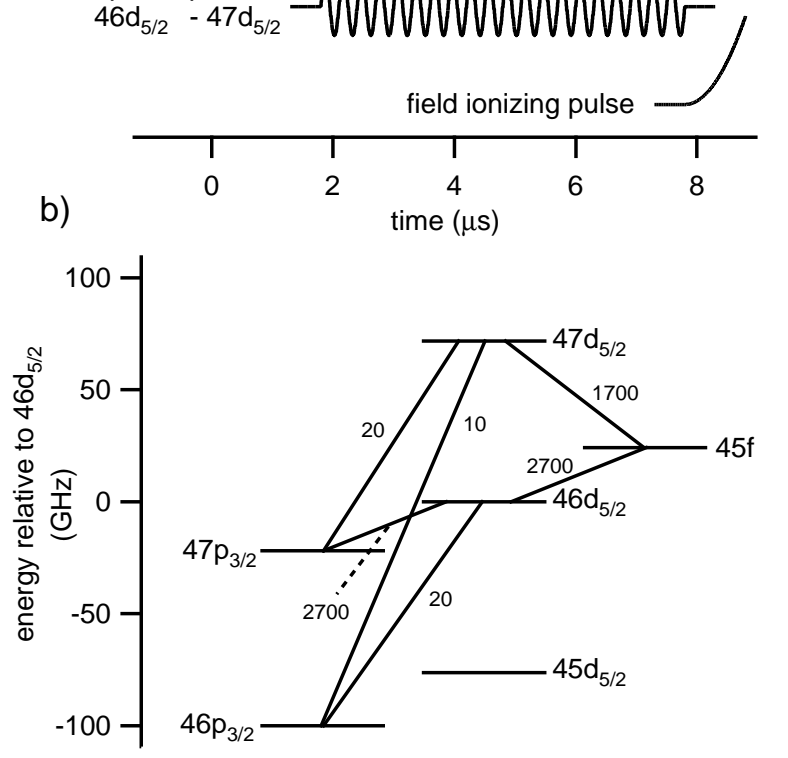

FIG. 1: a) Timing for experiment. There are delays of roughly 100 ns between the excitation laser pulse, transfer pulse, probe pulse and field ionizing pulse. This sequence is repeated at $10 \mathrm{~Hz}$ and is synchronized with the $60 \mathrm{~Hz} \mathrm{AC}$ power line. b) Energy levels of relevant states. The spectroscopic data is from Ref. 21]. The matrix elements magnitudes $\left|<n \ell_{j}\right| r\left|n^{\prime} \ell_{j^{\prime}}^{\prime}>\right|$ are shown between appropriate states, where $r$ is the radial coordinate of the Rydberg electron. These are expressed in atomic units, and were calculated using the techniques described by Zimmerman et al. 222].

\section{OBSERVATION OF THE RESONANT ELECTRIC DIPOLE-DIPOLE INTERACTION}

The interactions between ${ }^{85} \mathrm{Rb}$ Rydberg atoms were studied using the linewidths of the $46 d_{5 / 2}-47 d_{5 / 2}$ twophoton "probe" transition. Li et al. [21] have shown that two-photon transitions between states with identical $g_{J}$ factors, such as $46 d_{5 / 2}$ and $47 d_{5 / 2}$, show negligible broadening in the inhomogeneous magnetic field of a MOT. These transitions also have less sensitivity to electric fields than one-photon transitions.

The typical experimental sequence for a single shot is shown in Fig. 17). Depending on the particular situation, a "transfer pulse" may be used shortly after optical excitation to drive a fraction of the initially excited $46 d_{5 / 2}$ atoms into other states. In the presence of the inhomoge- neous magnetic field of the MOT any coherent superpositions formed by this pulse will rapidly dephase across the sample. Interatomic interactions are somewhat inhomogeneous due to the variation in spacings between atoms, and are also expected to cause appreciable dephasing. As a consequence the transfer pulse is viewed as redistributing population rather than creating superpositions.

The $46 d_{5 / 2}-47 d_{5 / 2}$ probe microwave frequency is scanned between shots. The power of this probe is adjusted so that power broadening is negligible, and in the limit of low Rydberg densities, the linewidths of this transition are entirely dominated by transform broadening. To analyze additional broadening due to interatomic effects, the spectra are fit to the transform limited lineshape for a square excitation pulse $\left(\operatorname{sinc}^{2}(\pi f T)\right)$ convolved with a Lorentzian of variable width $\delta \nu$. The widths of the fitted Lorentzian are then analyzed as a function of spatially averaged total Rydberg density (which is normally adjusted using the $480 \mathrm{~nm}$ excitation laser power).

As illustrated in Fig. 2] $\delta \nu$ converges to zero as the Rydberg density goes to zero. This indicates that the line broadening is an interatomic effect. At $1 \times 10^{7} \mathrm{~cm}^{-3} \mathrm{Ry}-$ dberg density, transferring one-half of the $46 d_{5 / 2}$ atoms to $47 p_{3 / 2}$ broadens the linewidth, increasing $\delta \nu$ from approximately $10 \mathrm{kHz}$ to $120 \mathrm{kHz}$ (the uncertainty in $\delta \nu$ is typically $\pm 7 \mathrm{kHz}$ ). This can be attributed to the resonant electric dipole-dipole interaction between atoms in these two states. Consider a pair of atoms labelled A and B. In the absence of interactions between these atoms, the state $\left|46 d_{5 / 2} m_{5 / 2}>_{\mathrm{A}}\right| 47 p_{3 / 2} m_{3 / 2}>_{\mathrm{B}}$ is energy degenerate with $\left|47 p_{3 / 2} m_{3 / 2}>_{\mathrm{A}}\right| 46 d_{5 / 2} m_{5 / 2}>_{\mathrm{B}}$. The dipole-dipole interaction breaks this degeneracy, leading to an energy splitting on the order of

$$
\Delta \nu_{d d} \approx \frac{\left|<46 d_{5 / 2}\right| r\left|47 p_{3 / 2}>\right|^{2}}{R_{\mathrm{AB}}^{3}}
$$

where $r$ is the separation of Rydberg electron from the ion-core, and $R_{\mathrm{AB}}$ is the typical interatomic spacing $[23$, 24.

The value of $\left|<46 d_{5 / 2}\right| r\left|47 p_{3 / 2}>\right|$ and other relevant radial transition dipole moments are indicated in Fig. 1). For instance, the dipole coupling between the $47 d_{5 / 2}$ and $47 p_{3 / 2}$ states is relatively weak compared to the coupling between the $46 d_{5 / 2}$ and $47 p_{3 / 2}$ states. Thus the broadening of the two-photon $46 d_{5 / 2}-47 d_{5 / 2}$ probe transition beyond the transform limit is dominated by dipole-dipole energy splittings in the initial state. As in Ref. 13], a simple estimate based on Eq. 22 is consistent with the observed broadening. The $1 / R_{\mathrm{AB}}^{3}$ scaling of the splittings implies a linear density dependence of the line broadening, which is clearly demonstrated in Fig. 2a).

The dipole-dipole coupling between $46 d_{5 / 2}$ and $45 f$ states is of comparable magnitude to that between $46 d_{5 / 2}$ and $47 p_{3 / 2}$ states (see Fig. [1). Consequently, transfer of a fraction of atoms to the $45 f$ state gives similar broadening (see Fig. 20). 
It is also straightforward to drive transitions to a nearby state which is not strongly dipole coupled to either the initial or final state of the two-photon probe transition. Based on the radial dipole matrix elements we would expect 4 orders of magnitude less broadening if $50 \%$ of the atoms were transferred to $46 p_{3 / 2}$ instead of $47 p_{3 / 2}$. As Fig. 2b) indicates, there is no detectable broadening of the probe transition in the presence of $46 p_{3 / 2}$ atoms.

These results are similar to those presented in Ref. 13] (only at one higher principal quantum number). There is a slight increase in linewidth of $\approx 20 \%$ in a mixture of $n d_{5 / 2}$ and $(n+1) p_{3 / 2}$ atoms (where $n=46$ here, and $n=45$ in Ref. 13]). The energy splittings $\Delta \nu_{d d}$ scale like $n^{4}$ due to the $n^{2}$ scaling of the transition dipole moments 16], and this accounts for about a $10 \%$ increase. We have also made changes in our Rydberg density estimate procedure, and these may also account for the discrepency. The more significant difference is in the linewidths obtained when no transfer pulse is present (see Fig. 2). There is roughly 3 times less broadening as compared to the data presented in Ref. [13].

The source of broadening in the case of no transfer pulse was proposed to be due to the collision of hot Rydberg atoms $(\approx 300 \mathrm{~K})$ with the cold Rydberg atoms [13]. These types of collisions have also been discussed in the context of cold plasma formation [25]. The hot atoms are excited in the same way as the cold trapped atoms. They make an undetectable contribution to the observed field ionization because the waiting period between excitation and extraction allows them to move to where they can't be detected.

Several experimental tests support the role of the hot Rydberg atoms in line broadening. To control the hot background Rb number density, the current through an alkali metal dispenser source is varied 26]. When the hot $\mathrm{Rb}$ background density is varied in this manner, the 480 $\mathrm{nm}$ excitation laser power should be adjusted to produce the same cold Rydberg density (compensating for the different trapped atom density). The time constant is relatively long for changes in background pressure with dispenser current (on the order of 10 minutes), so we can check independently of background $\mathrm{Rb}$ pressure whether thermal radiation or charged particles from the dispenser are responsible for broadening. At our sensitivity, no such effects were found.

With constant cold Rydberg density, it is found that $\delta \nu$ increases with hot Rb background density. This rules out the interactions between cold Rydberg atoms as being responsible for the broadening. However, at higher densities than those studied here, the nearly resonant interactions $46 d_{5 / 2} 46 d_{5 / 2} \leftrightarrow 48 p_{3 / 2} 44 f$ between cold Rydberg atoms will become increasingly important [12]. At a fixed hot Rb background density, $\delta \nu$ is proportional to $480 \mathrm{~nm}$ excitation laser power, which rules out the influence of unexcited hot atoms.

Not only does the hot Rydberg atom density increase with background $\mathrm{Rb}$ pressure, but so does the

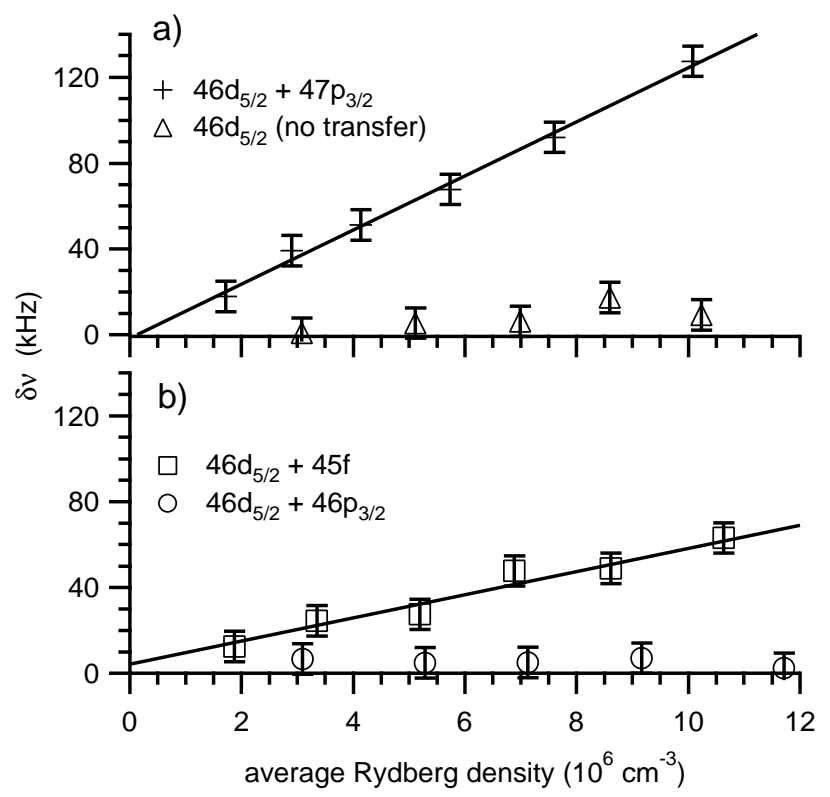

FIG. 2: Broadening of the $46 d_{5 / 2}-47 d_{5 / 2}$ probe transition as a function of average Rydberg density with and without transfer pulses. The transfer pulses create $50 \%$ mixtures, except in the case of the $46 d_{5 / 2}+45 f$ mixture, where only $30 \%$ of the total Rydberg atoms are transferred to the $45 f$ state. The straight lines are least squares fits. See the text for the definition of $\delta \nu$.

trapped atom density. This suggests a check to determine whether interactions with less excited cold atoms, or with hot Rydberg atoms from the vapor, are the cause of the increased broadening. If the cooling and trapping laser frequency is detuned to obtain less cold atoms, and the $480 \mathrm{~nm}$ laser power is increased to obtain the same cold Rydberg density, the observed linewidths increase, because more hot atoms are optically excited to Rydberg states. If less excited cold atoms were responsible for the broadening we would expect a decrease in linewidth.

In summary, the broadening $\delta \nu$ observed with no transfer pulse is due to the collision of hot Rydberg atoms with the cold Rydberg atoms we are studying. In the present work $\delta \nu$ in the absence of a transfer pulse is smaller than in Ref. [13] for at least two reasons. We are now working at lower dispenser currents (corresponding to lower background pressures) and using a $480 \mathrm{~nm}$ excitation source with significantly narrower bandwidth. The reduced bandwidth is expected to alter the ratio of cold to hot Rydberg atoms.

By working with a low hot Rb background pressure, and at $10^{7} \mathrm{~cm}^{-3}$ total Rydberg density, $\delta \nu$ is on the order of its experimental uncertainty $\pm 7 \mathrm{kHz}$. We have not yet established what the ultimate limit on the lowest $\delta \nu$ is, and thus the ultimate sensitivity of the probe transition to Rydberg interactions. However, the currently demonstrated sensitivity is vastly superior to what can readily be achieved with optical probes. 


\section{MAGNETIC FIELD MEASUREMENTS}

In this section we discuss the use of a single-photon microwave transition between Rydberg states to measure magnetic fields.

The MOT used to trap the ${ }^{85} \mathrm{Rb}$ atoms requires a magnetic quadrupole field [15]. In these experiments the field gradients in the three orthogonal directions are estimated to be 12,12 and $24 \mathrm{G} / \mathrm{cm}$, based on the coil geometry. The Rydberg cloud has a Gaussian profile with FWHM's of $0.190 \pm 0.015 \mathrm{~mm}$ in two dimensions (dictated by the $480 \mathrm{~nm}$ excitation laser) and $0.5 \pm 0.1 \mathrm{~mm}$ in the third dimension (dictated by the trap size). The largest dimension corresponds to the direction of the largest magnetic field gradient. This gives a root mean square (RMS) magnetic field of approximately $0.5 \mathrm{G}$. Since Zeeman shifts are typically on the order of $1 \mathrm{MHz} / \mathrm{G}$, and the dipoledipole interactions that we observe are roughly $100 \mathrm{kHz}$, it is apparent that the quadrupole field is a significant inhomogeneity, and it is desirable to turn this off. The current in the coils producing this field is shut off in $1.7 \mathrm{~ms}$, but significant eddy currents persist for much longer (see, for example, Ref. [27]). Excitation to Rydberg states occurs after a delay of $25 \mathrm{~ms}$, to allow the eddy currents to die down (see Fig. 3). As will be discussed, an upper bound can be placed on any remaining inhomogeneity.

The detuning and counterpropagating beam balance of the MOT lasers are optimized to minimize the reduction in cold atom density during the $25 \mathrm{~ms}$ delay. Immediately after selective field ionization is complete, the current to the quadrupole coils is turned back on to recapture cold atoms for the next shot.

Once the quadrupole field is turned off, a DC magnetic field may remain. Three pairs of orthogonal Helmholtz coils are used to null any preexisting field (due to the ionpump, earth etc...) and one of these coil pairs is used to produce a deliberate homogeneous field. The current in the coils is constant throughout the MOT collection, Rydberg excitation, and microwave probe cycle. When setting a non-zero homogeneous field, the quadrupole zero of the MOT will be shifted. As long as the quadrupole zero remains significantly within the overlap of the cooling and trapping beams, the trap will still function. The use of fields of up to $0.6 \mathrm{G}$ pointing in the direction with a field gradient of $12 \mathrm{G} / \mathrm{cm}$, shifts the zero by $0.5 \mathrm{~mm}$, which does not significantly influence MOT operation when using $780 \mathrm{~nm}$ beams with a FWHM of $4 \mathrm{~mm}$. Loss of the cold atoms in the $25 \mathrm{~ms}$ waiting period limits the DC bias fields which can be applied using this technique.

Although it is straightforward to calculate the fields due to the bias coils from their geometries, we do not $a$ priori know the stray magnetic field within the apparatus. To determine this, the one-photon microwave transition $34 s_{1 / 2}-34 p_{1 / 2}$ of ${ }^{85} \mathrm{Rb}$ is used. We work at a lower $n$ compared to where the dipole-dipole experiment is performed so that the hyperfine spacings are larger (these scale like $1 / n^{3}$, see Ref. [16]). To improve the sensitivity of the measurement it is desirable to use long microwave

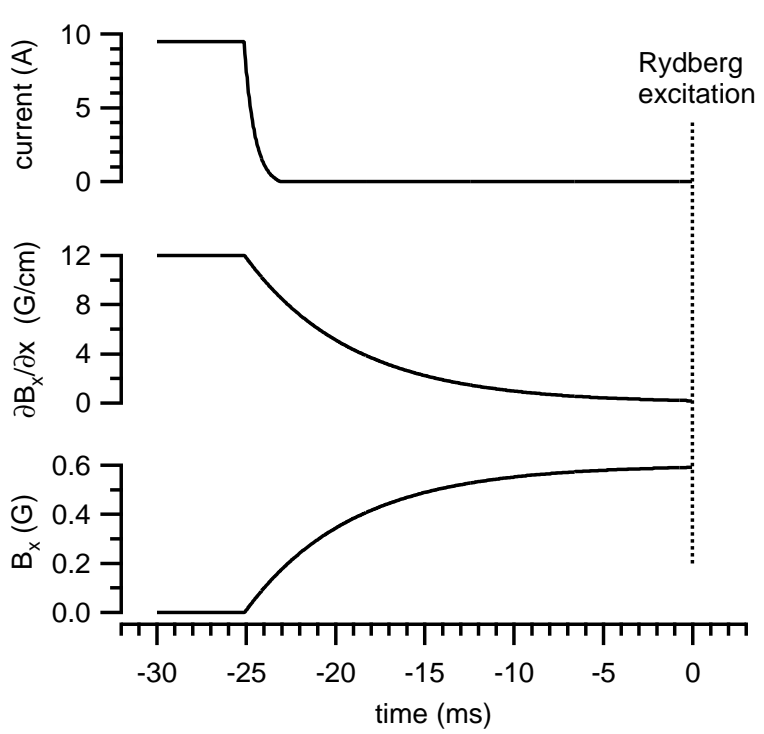

FIG. 3: Timing for experiments done in the presence of a homogeneous DC magnetic field. The quadrupole field coil current is cut to zero in $1.7 \mathrm{~ms}$. As the magnetic field gradient $\partial B_{x} / \partial x$ decays to zero, the field in the center of the trap $B_{x}$ gradually increases due to the presence of a non-zero homogeneous bias field. At $t=0$, excitation to Rydberg states and subsequent microwave probing takes place, as shown in Fig. 11 The gradient and bias field time constant was measured using Rydberg atom microwave spectroscopy (see text).

pulse lengths (for narrow linewidths). Redistribution of Rydberg state population due to thermal radiation, limits the microwave pulse length. Pulses of $36 \mu$ s are used.

The Zeeman splittings of the relevant levels are shown in Fig. 4 The $780 \mathrm{~nm}$ light used for optical excitation is nearly resonant with only the $5 s_{1 / 2} F=3$ to $5 p_{3 / 2} F=4$ transition. Since $F$ cannot change by more than one in a single photon transition, the $34 s_{1 / 2} F=2$ state is not excited in zero magnetic field, and only two microwave spectral lines are observable: $34 s_{1 / 2}, F=3$ to $34 p_{1 / 2}, F=2$ and $34 s_{1 / 2}, F=3$ to $34 p_{1 / 2}, F=3$ (see Fig. [5).

As a magnetic field is applied, this spectrum splits into many lines. To zero the magnetic field in a specific direction the following procedure is followed. A current is applied to a set of coils to produce a relatively large magnetic field (the precise value is unknown at this point). By scanning the microwave frequency, a spectrum with many lines can be observed (see Fig. 5). Then we determine the current which produces the same field magnitude in the opposite direction, by trying to reproduce the spectral line positions. The average of these two currents corresponds to zero field projection along this axis. Using this procedure all three orthogonal axes can be zeroed independently. Since the change in magnetic field with current can be estimated from coil geometry, the precision to which the average currents can be determined can be directly related to the precision to which the fields can be compensated. We have zeroed the magnetic field along each axis to within $\pm 6 \mathrm{mG}$ using this technique. 


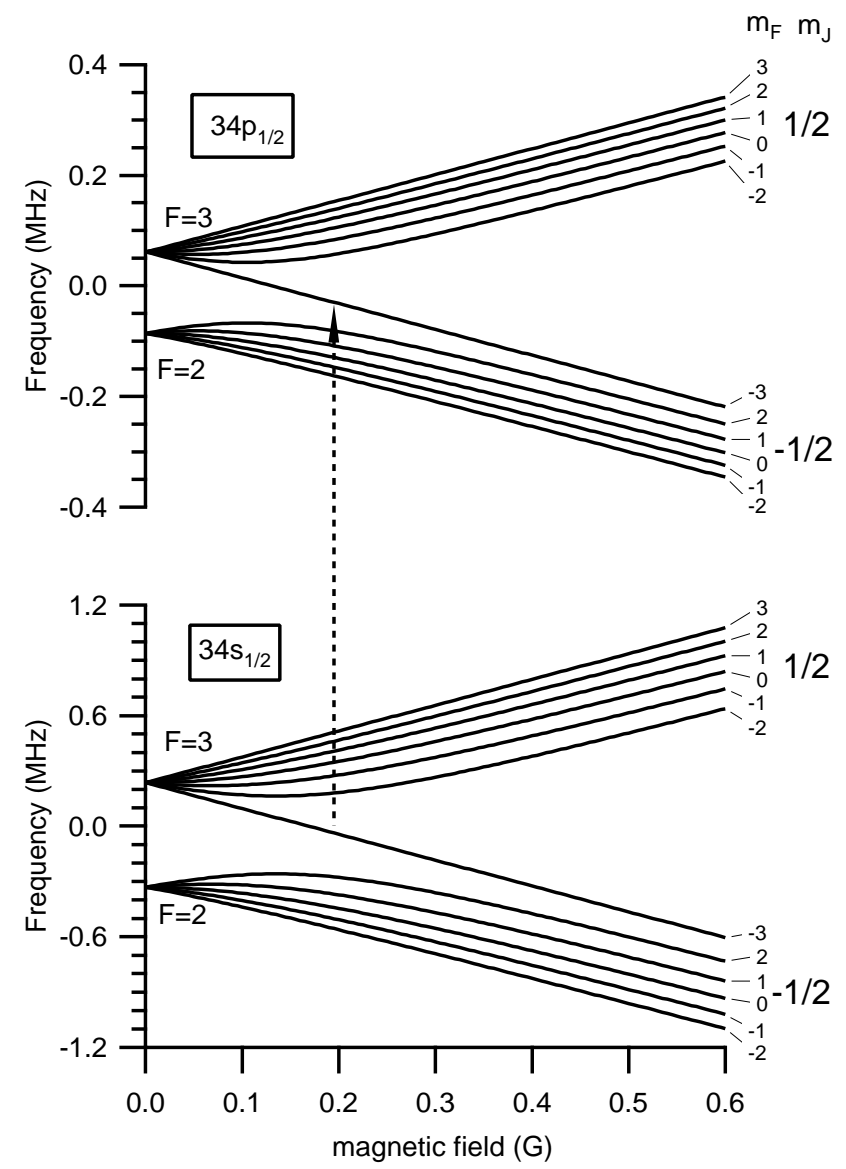

FIG. 4: Calculated Zeeman structure of the $34 s_{1 / 2}$ and $34 p_{1 / 2}$ levels of ${ }^{85} \mathrm{Rb}$ using the Breit-Rabi formulae [28] (the $m_{J}$ labelling is approximate). Please note the different vertical scalings. The two hyperfine splittings are chosen to match the spectral lines in Fig. 5 ( $0.567 \mathrm{MHz}$ for $34 s_{1 / 2}$, and $0.147 \mathrm{MHz}$ for $\left.34 p_{1 / 2}\right)$. The arrow labels the $34 s_{1 / 2}\left(m_{F}=-3\right)$ to $34 p_{1 / 2}\left(m_{F}=-3\right)$ line, which is used for magnetic field calibration (see text).

To deliberately create magnetic fields in a specific direction, one of the coil currents was adjusted away from its "zero" value. The change in magnetic field with coil current can be estimated from the coil geometry. However, the line positions in the $34 s_{1 / 2}-34 p_{1 / 2}$ spectra (see Fig. 5) can be used to directly measure the field. The shifting of energies is given by the Breit-Rabi formulae (see, for example Ref. 28 ). In general, line positions are dependent on the magnetic field, and the hyperfine splitting of both the $34 s_{1 / 2}$ and $34 p_{1 / 2}$ states. The $34 s_{1 / 2}$ hyperfine spacing is not known a priori. However, there is a particular line (marked with arrows in Fig. 5), corresponding to the transition from $34 s_{1 / 2}\left(m_{F}=-3\right)$ to $34 p_{1 / 2}\left(m_{F}=-3\right)$ whose shift in position with magnetic field does not depend on the hyperfine spacing. The tran-

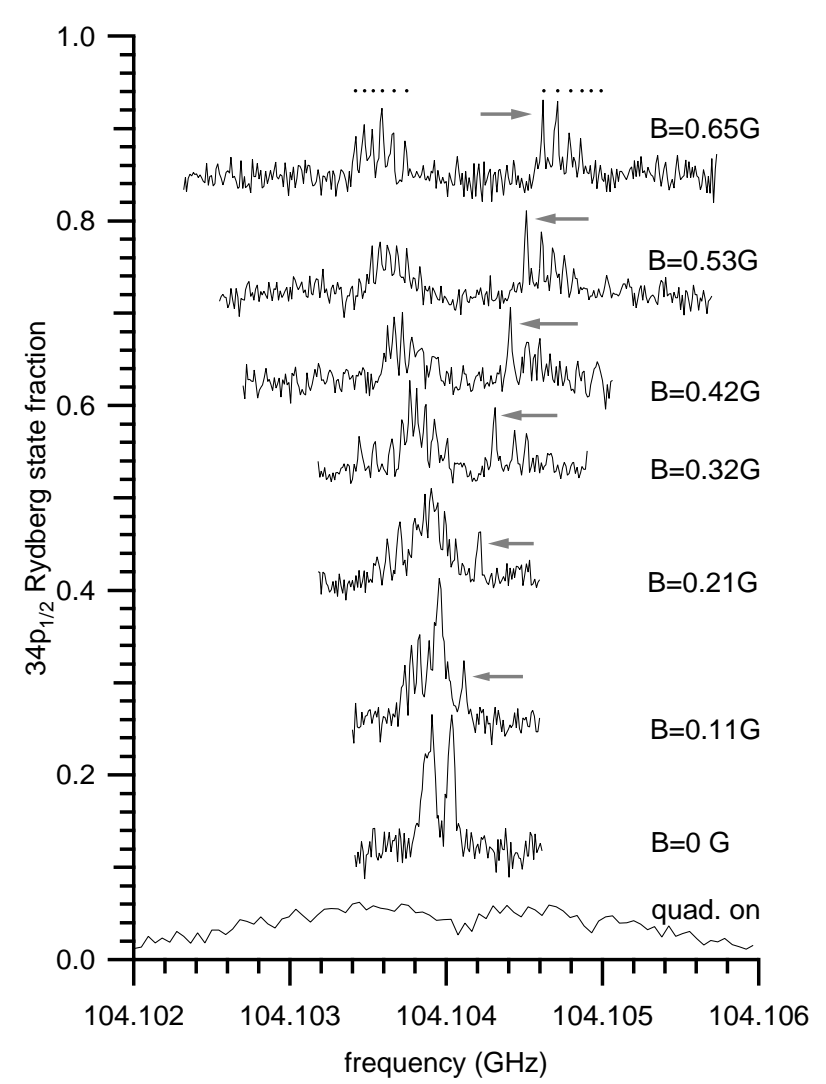

FIG. 5: The ${ }^{85} \mathrm{Rb} 34 s_{1 / 2}$ to $34 p_{1 / 2}$ microwave spectra taken with different DC magnetic fields (top 7 traces) and with the MOT quadrupole field present (bottom trace). Spectra are offset vertically for clarity. The calculated line positions of the $\Delta m_{F}=0$ transitions using the energy levels from Fig. 4 are shown for the $0.65 \mathrm{G}$ data (the microwave polarization vector is in same direction as the applied magnetic field). The arrow labels the $34 s_{1 / 2}\left(m_{F}=-3\right)$ to $34 p_{1 / 2}\left(m_{F}=-3\right)$ line, which is used for magnetic field calibration (see text).

sition energy for this line is $(2 / 3) \mu_{B} B$, where $\mu_{B}$ is the Bohr magneton and $B$ is the magnetic field. As apparent in Fig. [5 this line is convenient as it is readily identifable in the spectra. By tabulating the position of this spectral line as a function of coil current and fitting a straight line, the magnetic field as a function of coil current can be determined, without knowledge of the $34 s_{1 / 2}$ hyperfine splitting. This provides a precise determination of the magnetic field $( \pm 10 \mathrm{mG})$. The dipole-dipole interaction is then studied under these calibrated magnetic field conditions.

It is desirable to know the inhomogeneity in the magnetic field when the experiment is performed. The bottom trace in Fig. [5]shows inhomogeneous broadening due to the quadrupole field when the coil current is not shut off. By varying the time between between shutting the current off and excitation of the atoms to Rydberg states (see Fig. 3) we can observe the decay of this inhomogeneity using the spectral widths. The decay is observed to be exponential with a time constant of $6 \pm 1 \mathrm{~ms}$. A gauss- 
meter on the outside of the vacuum chamber, placed as close as possible to the experimental region (within $15 \mathrm{~cm}$ ) shows a similar time constant for the change in magnetic field: $6.5 \pm 0.5 \mathrm{~ms}$.

At the start of this section, the RMS magnetic field due to the quadrupole field was calculated to be $0.5 \mathrm{G}$. Based on the exponential decay of the gradient, $25 \mathrm{~ms}$ after the coil currents are shut-off we would expect this to decay to $8 \mathrm{mG}$. This inhomogeneity is insignificant in the dipole-dipole interaction experiments discussed in the next section.

The widths of the resolved $34 s_{1 / 2}\left(m_{F}=-3\right)$ to $34 p_{1 / 2}\left(m_{F}=-3\right)$ spectral lines in Fig. 5] put an upper bound on the magnetic field inhomogeneity. The lines are observed to have a linewidth of $30 \mathrm{kHz}$. The transform limited linewidth for a $36 \mu$ s long pulse is approximately $25 \mathrm{kHz}$. If as a worst case we assume that the linewidth contributions combine in quadrature, the residual broadening due to magnetic field inhomogeneity and other mechanisms is $17 \mathrm{kHz}$. This gives a rough upper bound of $17 \mathrm{mG}$ on the magnetic field inhomogeneity, which is consistent with the estimate given in the previous paragraph.

\section{SUPPRESSION OF THE RESONANT ELECTRIC DIPOLE-DIPOLE INTERACTION DUE TO A MAGNETIC FIELD}

Using the techniques described in the previous section, cold Rydberg atom interactions can be studied in the presence of homogeneous, calibrated magnetic fields. The timing of the experiment remains the same as in Fig. 17). However, the current to the quadrupole coils is shut-off $25 \mathrm{~ms}$ prior to photoexcitation (see Fig. 3). During this waiting period, the cold atom cloud dimensions expand. At each different applied magnetic field value, the counterpropagating beam powers are re-optimized to minimize expansion. When this is done, it is found that trap expansion is independent of DC magnetic field to within $10 \%$ for fields less than $0.6 \mathrm{G}$, and linewidth broadening can be studied at a fixed Rydberg density as a function of magnetic field.

The linewidth broadening due to interatomic effects, $\delta \nu$ is obtained in exactly the same manner as described in Section III In the limit of low densities $\delta \nu$ approaches zero for all DC magnetic fields, indicating that it is due to interatomic interactions. At an average Rydberg density of $8.0 \times 10^{6} \mathrm{~cm}^{-3}$ we have found that conversion of $50 \%$ of the $46 d_{5 / 2}$ atoms to $47 p_{3 / 2}$ increases $\delta \nu$ from $10 \mathrm{kHz}$ to $110 \mathrm{kHz}$ at approximately $0.04 \mathrm{G}$. As Fig. 6] indicates, increasing the magnetic field reduces $\delta \nu$. The presence of a DC magnetic field suppresses the resonant dipole-dipole interaction between atoms. Essentially the magnetic field spoils some of the unperturbed atom energy degeneracies, weakening the resonant interaction.

To discuss this in detail, we consider the dipole-dipole

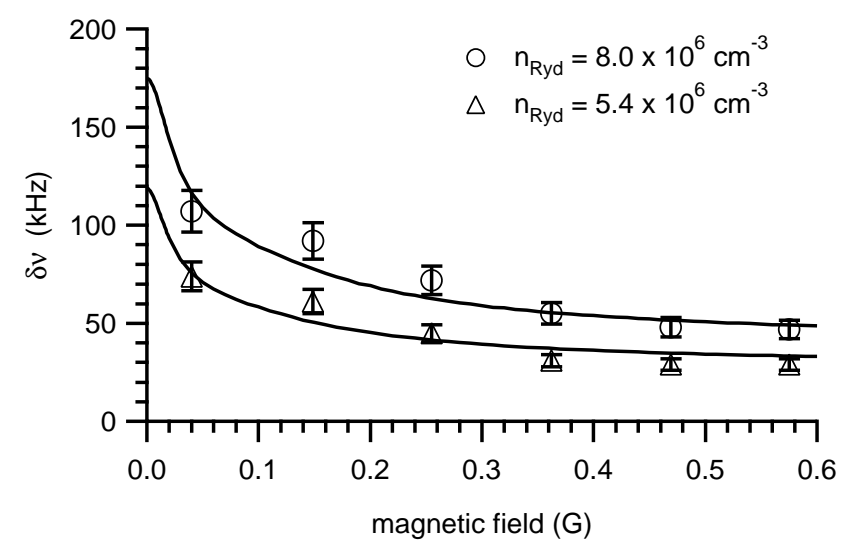

FIG. 6: Broadening of the $46 d_{5 / 2}-47 d_{5 / 2}$ probe transition in the presence of $50 \% 47 p_{3 / 2}$ atoms as a function of magnetic field strength. Two different total Rydberg number densities are shown. The solid lines are calculations (see text for details).

interaction operator:

$$
\hat{V}_{d d}=\frac{\vec{\mu}_{\mathrm{A}} \cdot \vec{\mu}_{\mathrm{B}}-3\left(\vec{\mu}_{\mathrm{A}} \cdot \vec{n}\right)\left(\vec{\mu}_{\mathrm{B}} \cdot \vec{n}\right)}{R_{\mathrm{AB}}^{3}}
$$

where $\vec{\mu}_{\mathrm{A}}$ and $\vec{\mu}_{\mathrm{B}}$ are the electric dipole matrix element operators evaluated on each atom, $\vec{n}$ is the unit vector pointing between the atoms, and $R_{\mathrm{AB}}$ is the separation of the two atoms.

The tensor product of the separated, non-interacting atom energy eigenstates can be used as a basis set for the evaluation of $\hat{V}_{d d}$. The axis of quantization for the projection of the angular momentum of each atom is chosen to point in the same direction as the magnetic field. This is in contrast to the more conventional choice for interatomic interactions, where the quantization axis is chosen along $\vec{n}$ (see, for example, Ref. 29]). This choice complicates the evaluation of the matrix elements of $\hat{V}_{d d}$, but simplifies the evaluation of the Zeeman part of the Hamiltonian.

If we label each atom as $\mathrm{A}$ and $\mathrm{B}$, in zero magnetic field with no dipole-dipole interaction, all 24 states of the form $|1>=| 46 d_{5 / 2} m_{j, \mathrm{~A} 1}>_{\mathrm{A}} \mid 47 p_{3 / 2} m_{j, \mathrm{~B} 1}>_{\mathrm{B}}$ are energy degenerate with the 24 states of the form $|2>=| 47 p_{3 / 2} m_{j, \mathrm{~A} 2}>_{\mathrm{A}}$ $\mid 46 d_{5 / 2} m_{j, \mathrm{~B} 2}>_{\mathrm{B}}$. It is this degeneracy which is responsible for the resonant electric dipole-dipole line broadening.

Magnetic fields lift some of this degeneracy through the Zeeman effect. If $\left|\psi_{b}\right\rangle$ is a basis set vector of the form given above, then:

$$
<\psi_{b}\left|\hat{V}_{Z}\right| \psi_{b}>=\left(g_{j \mathrm{~A}} m_{j \mathrm{~A}}+g_{j \mathrm{~B}} m_{j \mathrm{~B}}\right) \mu_{B} B
$$

where $g_{j}$ is given by the standard formula $\left(g_{j}=4 / 3\right.$ for the ${ }^{2} p_{3 / 2}$ states, and $g_{j}=6 / 5$ for the ${ }^{2} d_{5 / 2}$ states [28]). In this basis set, with the axis of quantization along the direction of the magnetic field, there are no off-diagonal elements of $\hat{V}_{Z}$. 
TABLE I: Non-zero matrix elements $\left\langle 2\left|\hat{V}_{d d}\right| 1\right\rangle$ where $|1\rangle=$ $\left|46 d_{5 / 2} m_{j}=1 / 2>_{\mathrm{A}}\right| 47 p_{3 / 2} m_{j}=1 / 2>_{\mathrm{B}}$ and $|2\rangle=\mid 47 p_{3 / 2} m_{j, \mathrm{~A}}>_{\mathrm{A}}$ $\mid 46 d_{5 / 2} m_{j, \mathrm{~B}}>_{\mathrm{B}}$, and $R_{\mathrm{AB}}=28.5 \mu \mathrm{m}$. The axis for angular momentum quantization is in the direction of $\vec{B}$ (see text). The dipole moments $\vec{\mu}_{\mathrm{A}}$ and $\vec{\mu}_{\mathrm{B}}$ in Eq. 3 are evaluated using the techniques described in Ref. 22]. The Zeeman energy shifts of the $\mid 2>$ states relative to the $\mid 1>$ state are: $\mu_{\mathrm{mag}, 2}-$ $\mu_{\text {mag, } 1}=\left[<2\left|\hat{V}_{Z}\right| 2>-<1\left|\hat{V}_{Z}\right| 1>\right] / B$ (calculated using Eq. 4).

\begin{tabular}{r|r|c|r|c|c}
\hline \hline \multicolumn{3}{|c|}{$\mid 2>$} & \multicolumn{2}{|c|}{$\left.<2\left|\hat{V}_{d d}\right| 1\right\rangle(\mathrm{kHz})$} & $\mu_{\mathrm{mag}, 2}-\mu_{\mathrm{mag}, 1}$ \\
\cline { 2 - 4 }$m_{j, \mathrm{~A}}$ & $m_{j, \mathrm{~B}}$ & $m_{j, \text { tot }}$ & $\vec{n} \| \vec{B}$ & $\vec{n} \perp \vec{B}$ & $(\mathrm{kHz} / \mathrm{G})$ \\
\hline$-1 / 2$ & $-1 / 2$ & -1 & 0 & 55 & -3547 \\
$3 / 2$ & $-1 / 2$ & 1 & 21 & -11 & 187 \\
$1 / 2$ & $1 / 2$ & 1 & -147 & 74 & 0 \\
$-1 / 2$ & $3 / 2$ & 1 & 52 & -26 & -187 \\
$3 / 2$ & $3 / 2$ & 2 & 0 & 45 & 3547 \\
\hline \hline
\end{tabular}

Once a magnetic field is applied only pairs of states for which $m_{j, \mathrm{~A} 1}=m_{j, \mathrm{~B} 2}$ and $m_{j, \mathrm{~B} 1}=m_{j, \mathrm{~A} 2}$, are degenerate. As an example, consider the state $\mid 46 d_{5 / 2} m_{j}=1 / 2>_{\mathrm{A}}$ $\mid 47 p_{3 / 2} m_{j}=1 / 2>_{\mathrm{B}}$. This is coupled by $\hat{V}_{d d}$ to several states, as shown in Table \ These states are all energy degenerate in the absence of a magnetic field and interactions. Once a magnetic field is applied only one coupled state has an identical Zeeman shift and remains degenerate: $\left|47 p_{3 / 2} m_{j}=1 / 2>_{\mathrm{A}}\right| 46 d_{5 / 2} m_{j}=1 / 2>_{\mathrm{B}}$. Since the application of a magnetic field lowers the energy degeneracy, we expect less line broadening since some of the dipole-dipole couplings are no longer resonant.

A quantitative treatment of the resulting linewidth suppression will now be considered. The essence of this treatment is the numerical diagonalization of a Hamiltonian using a basis set of two atom states - the $24+24=48$ states mentioned above. All of these 48 states are energy degenerate in the absence of a magnetic field and dipoledipole interactions, so the simplified Hamiltonian is

$$
\hat{H}=\hat{V}_{Z}+\hat{V}_{d d}
$$

This corresponds to a 48 by 48 matrix for fixed values of $\vec{n}, R_{\mathrm{AB}}$ and $B$. The matrix elements of $\hat{V}_{d d}$ require the transition dipole moments $\vec{\mu}_{\mathrm{A}}$ and $\vec{\mu}_{\mathrm{B}}$ (see Eq. 31), which can be evaluated using numerical integration of the Rydberg electron wave-functions [22]. The matrix elements of $\hat{V}_{Z}$ are obtained from Eq. 4 The resulting Hamiltonian matrix may be numerically diagonalized, yielding a set of energy eigenvalues $E_{i}$ and corresponding eigenvectors $\left|\psi_{i}\right\rangle$.

As has been discussed, the two-photon probe transition is not sensitive to the Zeeman effect 21]. Thus for a correspondence with the spectral transition energies, the Zeeman energy contribution is subtracted from each eigenvalue:

$$
E_{c i}=E_{i}-<\psi_{i}\left|\hat{V}_{Z}\right| \psi_{i}>
$$

This heuristic expression is based on the observation that the probe linewidth is independent of magnetic field in the absence of interatomic effects. An exact treatment would take into account relative linestrengths in the twophoton probe transition.

The corrected energies $E_{c i}$ may be computed as a function of magnetic field magnitude for a fixed $R_{\mathrm{AB}}$ and relative orientation of $\vec{n}$ and $\vec{B}$. The distribution of the corrected energies dictates the linewidth broadening. If there was no dipole-dipole interaction these would all be zero. The spread in the corrected energies decreases with magnetic field - the magnetic field suppresses the dipoledipole interaction. To quantify this with a single number, we have computed the root mean square (RMS) corrected energy $\left\langle E_{c i}^{2}\right\rangle^{1 / 2}$ as a function of magnetic field for $\vec{n} \perp \vec{B}$ and $\vec{n} \| \vec{B}$ at a fixed value of $R_{\mathrm{AB}}$ (see Fig. (7).

The qualititative difference in the suppression for $\vec{n} \perp$ $\vec{B}$ and $\vec{n} \| \vec{B}$ may be understood from the difference in the selection rules for $\hat{V}_{d d}$ in these two cases. When $\vec{n}$ is parallel to the quantization axis $(\vec{B})$, the dipole-dipole interaction $\hat{V}_{d d}$ does not couple states of different $m_{j \text {,tot }}$, where $m_{j, \text { tot }}=m_{j, \mathrm{~A}}+m_{j, \mathrm{~B}}$. This is a consequence of the invariance of $\hat{V}_{d d}$ to rotations about $\vec{n}$. However the same selection rule does not exist when $\vec{n}$ is perpendicular to the quantization axis (see, for example, Table @). Due to the similarity in $g_{j}$ factors for the ${ }^{2} p_{3 / 2}$ and ${ }^{2} d_{5 / 2}$ states $(4 / 3$ vs. $6 / 5)$ the Zeeman shifts for states of the same $m_{j \text {,tot }}$ are quite similar when compared to states of different $m_{j \text {,tot }}$ (see the last column of Table【). Therefore the degeneracy between states coupled by $\hat{V}_{d d}$ with $\vec{n} \| \vec{B}$ is spoiled at much higher fields than for $\vec{n} \perp \vec{B}$. If Fig. 7 is extended to higher fields, then $\left\langle E_{c i}^{2}>^{1 / 2}\right.$ for $\vec{n} \| \vec{B}$ eventually drops to $67 \mathrm{kHz}$.

In the sample both $\vec{n}$ and $R_{\mathrm{AB}}$ are distributed randomly. The nearest neighbor probability distribution is given by [30]:

$$
\frac{d P\left(R_{\mathrm{AB}}\right)}{d R_{\mathrm{AB}}}=4 \pi R_{\mathrm{AB}}^{2} n_{\mathrm{int}} \exp \left(-4 \pi R_{\mathrm{AB}}^{3} n_{\mathrm{int}} / 3\right) .
$$

The number density $n_{\text {int }}$ in this expression is half of the total Rydberg density - as half of the atoms resonantly interact with the other half in an even mixture of $46 d_{5 / 2}$ and $47 p_{3 / 2}$ atoms. Using this expression and random orientations of $\vec{n}$, we have calculated the probability distribution of the corrected eigenvalues. These distributions were convolved with a $\operatorname{sinc}^{2}(\pi f T)$ lineshape $(T=6 \mu s)$ and fitted in the same manner as the experimental data (using the $\operatorname{sinc}^{2}(\pi f T)$ lineshape convolved with a Lorentzian of adjustable width $\delta \nu$ ).

To make a comparison with the experimental observations, the calculations must be done at a specific number density. As discussed, our experimental density determination is uncertain by almost a factor of 2 . Therefore the density used in the calculations is adjusted for the best fit to the data. This is the only adjustable parameter in the calculations, and we use the same adjustment factor for both densities in Fig. [6] Specifically, at the 


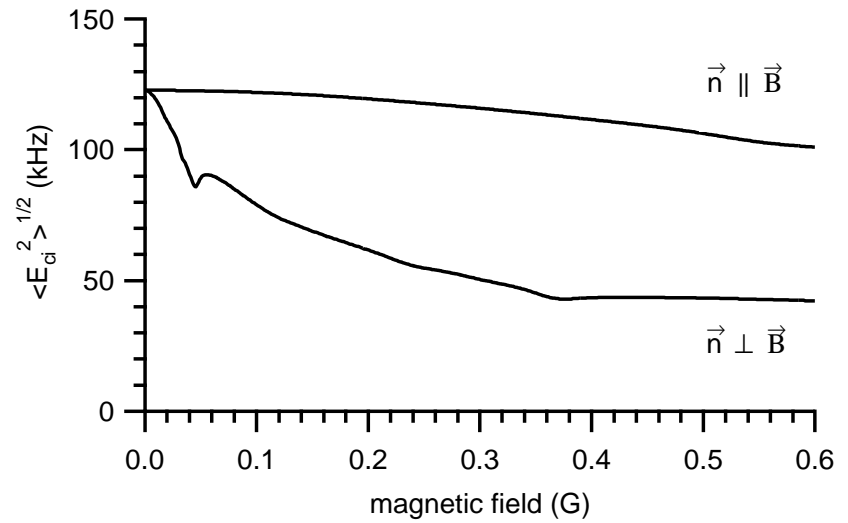

FIG. 7: The calculated RMS of the corrected energy eigenvalues $E_{c i}$ due to the electric dipole-dipole interaction between $46 d_{5 / 2}$ and $47 p_{3 / 2}$ atoms, as a function of magnetic field strength (see text). The interatomic separation is $R_{\mathrm{AB}}=$ $28.5 \mu \mathrm{m}$, the most probable resonantly interacting neighbor distance at a total Rydberg density of $1.4 \times 10^{7} \mathrm{~cm}^{-3}$. Calculations are shown for two different relative orientations of the magnetic field $\vec{B}$, and the unit vector pointing between the two atoms $\vec{n}$.

higher density the calculations are performed at a constant Rydberg density of $1.4 \times 10^{7} \mathrm{~cm}^{-3}$, whereas the spatially averaged experimental density is estimated to be $(0.8 \pm 0.5) \times 10^{7} \mathrm{~cm}^{-3}$. The discrepency may be an indication of the importance of multi-body interactions, which are expected to enhance the interactions over a simple binary model.

The calculations shown in Fig. 6 predict a reduction in linewidths with magnetic field comparable to the observations. However the agreement is not perfect. In particular, the experimental results appear to extrapolate to a lower linewidth at zero magnetic field. There are several possible reasons for this. Hyperfine structure has been neglected. This is expected to be especially important at low magnetic fields where the Zeeman shifts are smaller than the hyperfine splitting. In addition, stray electric fields break degeneracies between states of different $\left|m_{j}\right|$, and this is also expected to suppress linewidth.

The distribution of energy eigenvalues of the two atom system has been used to obtain linewidths - but linestrengths for the different transitions should be accounted for. In addition, not all of the eigenstates will be equally populated by the excitation scheme.

Perhaps the most significant problem with the theoretical model is the assumption of binary interactions: only pairs of atoms interact. Experimental observations of res- onant energy transfer between cold Rydberg atoms have demonstrated that this picture is not complete [1, 11]. Taking these into account would require a much more sophisticated approach [4, 31].

\section{CONCLUDING REMARKS}

The magnetic field induced partial suppression of the resonant electric dipole-dipole interaction between cold Rydberg atoms has been observed, and a quantitative model of this phenomena has been presented. This model could be tested in more detail. A "one-dimensional sample" has recently been successfully employed to determine the influence of the relative orientation of an electric field with $\vec{n}$ on Rydberg dipole-dipole interactions [32]. By focusing the Rydberg state excitation laser more tightly, so that the average interatomic separation is larger than the beam waist, the sample becomes one dimensional [32]. This gives a preferred direction to $\vec{n}$ in the lab-frame, and thus makes it possible to study interactions with $\vec{B} \perp \vec{n}$ or $\vec{B} \| \vec{n}$. As Fig. 7 indicates, there should be a dramatic difference between these two cases, which would provide a useful check.

Homogeneous DC magnetic fields cannot completely remove the energy degeneracies responsible for the resonant dipole-dipole interaction. For example, in the absence of interactions $\left|46 d_{5 / 2} m_{5 / 2}>_{\mathrm{A}}\right| 47 p_{3 / 2} m_{3 / 2}>_{\mathrm{B}}$ is energy degenerate with $\left|47 p_{3 / 2} m_{3 / 2}>_{\mathrm{A}}\right| 46 d_{5 / 2} m_{5 / 2}>_{\mathrm{B}}$ independent of magnetic field. For complete suppression of the dipole-dipole interaction it is interesting to consider the use of magnetic field gradients. By making the energy levels of the atoms depend on position, the resonance conditions can be completely spoiled. Strong magnetic field gradients have recently been used in electron spin resonance (ESR) to suppress spin diffusion due to the magnetic dipolar flip-flop interaction [33]. An analogous experiment could be performed in the case of resonant electric dipole-dipole interactions. The required field gradient is modest. For example, in the present work we typically have $100 \mathrm{kHz}$ interaction strengths at interatomic separations of $30 \mu \mathrm{m}$. With Zeeman shifts typically being on the order of $1 \mathrm{MHz} / \mathrm{G}$, field gradients of roughly $50 \mathrm{G} / \mathrm{cm}$ should give significant supression. These gradients are readily achievable - in magnetic microtraps for example 34]. Strong magnetic field gradients could be useful for suppression of resonant dipole-dipole interactions in situations where they are undesirable.

It is a pleasure to acknowledge discussions with T. F. Gallagher. We thank M. Fedorov, D. Vagale, and J. C. T. Martin for assistance. This work was supported by NSERC, CFI, and OIT.
[1] W. R. Anderson, J. R. Veale, and T. F. Gallagher, Phys. Rev. Lett. 80, 249 (1998).
[2] I. Mourachko, D. Comparat, F. de Tomasi, A. Fioretti, P. Nosbaum, V. M. Akulin, and P. Pillet, Phys. Rev. 
Lett. 80, 253 (1998).

[3] W. Li et al., Phys. Rev. A 70, 042713 (2004).

[4] F. Robicheaux, J. V. Hernández, T. Topçu, and L. D. Noordam, Phys. Rev. A 70, 042703 (2004).

[5] D. Jaksch, J. I. Cirac, P. Zoller, S. L. Rolston, R. Côté, and M. D. Lukin, Phys. Rev. Lett. 85, 2208 (2000).

[6] M. D. Lukin, M. Fleischhauer, R. Cote, L. M. Duan, D. Jaksch, J. I. Cirac, and P. Zoller, Phys. Rev. Lett. 87, 037901 (2001).

[7] M. Saffman and T. G. Walker, Phys. Rev. A 66, 065403 (2002).

[8] D. Tong, S. M. Farooqi, J. Stanojevic, S. Krishnan, Y. P. Zhang, R. Côté, E. E. Eyler, and P. L. Gould, Phys. Rev. Lett. 93, 063001 (2004).

[9] K. Singer, M. Reetz-Lamour, T. Amthor, L. G. Marcassa, and M. Weidemüller, Phys. Rev. Lett. 93, 163001 (2004).

[10] I. I. Ryabtsev, D. B. Tretyakov, and I. I. Beterov, J. Phys. B: At. Mol. Opt. Phys. 38, S421 (2005).

[11] I. Mourachko, W. Li, and T. F. Gallagher, Phys. Rev. A 70, 031401(R) (2004).

[12] W. Li, P. J. Tanner, and T. F. Gallagher, Phys. Rev. Lett. 94, 173001 (2005).

[13] K. Afrousheh, P. Bohlouli-Zanjani, D. Vagale, A. Mugford, M. Fedorov, and J. D. D. Martin, Phys. Rev. Lett. 93, 233001 (2004).

[14] I. Lesanovsky and P. Schmelcher, Phys. Rev. Lett. 95, 053001 (2005).

[15] C. Monroe, W. Swann, H. Robinson, and C. Wieman, Phys. Rev. Lett. 65, 1571 (1990).

[16] T. F. Gallagher, Rydberg Atoms (Cambridge University Press, 1994).

[17] B. K. Teo, D. Feldbaum, T. Cubel, J. R. Guest, P. R.
Berman, and G. Raithel, Phys. Rev. A 68, 053407 (2003).

[18] A. Osterwalder and F. Merkt, Phys. Rev. Lett. 82, 1831 (1999).

[19] Y. Suzaki and A. Tachibana, Appl. Opt. 14, 2809 (1975).

[20] G. W. Fraser, Int. J. Mass Spectrom. 215, 13 (2002).

[21] W. Li, I. Mourachko, M. W. Noel, and T. F. Gallagher, Phys. Rev. A 67, 052502 (2003).

[22] M. L. Zimmerman, M. G. Littman, M. M. Kash, and D. Kleppner, Phys. Rev. A 20, 2251 (1979).

[23] A. Fioretti, D. Comparat, C. Drag, T. F. Gallagher, and P. Pillet, Phys. Rev. Lett. 82, 1839 (1999).

[24] W. R. Anderson, M. P. Robinson, J. D. D. Martin, and T. F. Gallagher, Phys. Rev. A 65, 063404 (2002).

[25] M. P. Robinson, B. L. Tolra, M. W. Noel, T. F. Gallagher, and P. Pillet, Phys. Rev. Lett. 85, 4466 (2000).

[26] J. Fortagh, A. Grossmann, T. W. Hänsch, and C. Zimmermann, J. Appl. Phys. 84, 6499 (1998).

[27] C. J. Dedman, K. G. H. Baldwin, and M. Colla, Rev. Sci. Instrum. 72, 4055 (2001).

[28] H. G. Kuhn, Atomic Spectra (Longmans, London, 1962).

[29] R. Santra and C. H. Greene, Phys. Rev. A 67, 062713 (2003).

[30] S. Chandrasekhar, Rev. Mod. Phys. 15, 1 (1943).

[31] J. S. Frasier, V. Celli, and T. Blum, Phys. Rev. A 59, 4358 (1999).

[32] T. J. Carroll, K. Claringbould, A. Goodsell, M. J. Lim, and M. W. Noel, Phys. Rev. Lett. 93, 153001 (2004).

[33] R. Budakian, H. J. Mamin, and D. Rugar, Phys. Rev. Lett. 92, 037205 (2004).

[34] R. Folman, P. Krüger, J. Schmiedmayer, J. Denschlag, and C. Henkel, Adv. At. Mol. Opt. Phys. 48, 263 (2002). 